Musées, Patrimoine et Culture scientifiques et techniques

$192 \mid 2020$

novembre-décembre 2020

\title{
Le muséum sur roues : un engagement auprès et pour les publics
}

\section{Sandrine Amann et Alain Faber}

\section{OpenEdition \\ Journals}

Édition électronique

URL : https://journals.openedition.org/ocim/4178

DOI : $10.4000 /$ ocim. 4178

ISSN : 2108-646X

Éditeur

OCIM

Édition imprimée

Date de publication : 1 novembre 2020

Pagination : 28-35

ISSN : 0994-1908

Référence électronique

Sandrine Amann et Alain Faber, « Le muséum sur roues : un engagement auprès et pour les publics », La Lettre de I'OCIM [En ligne], 192 | 2020, mis en ligne le 01 novembre 2021, consulté le 11 décembre 2021. URL : http://journals.openedition.org/ocim/4178 ; DOI : https://doi.org/10.4000/ocim.4178

Ce document a été généré automatiquement le 11 décembre 2021.

Tous droits réservés 


\section{Le muséum sur roues : un engagement auprès et pour les publics}

Sandrine Amann et Alain Faber

Le Natur Mobil est principalement destiné aux jeunes scolaires de 4 à 11 ans.

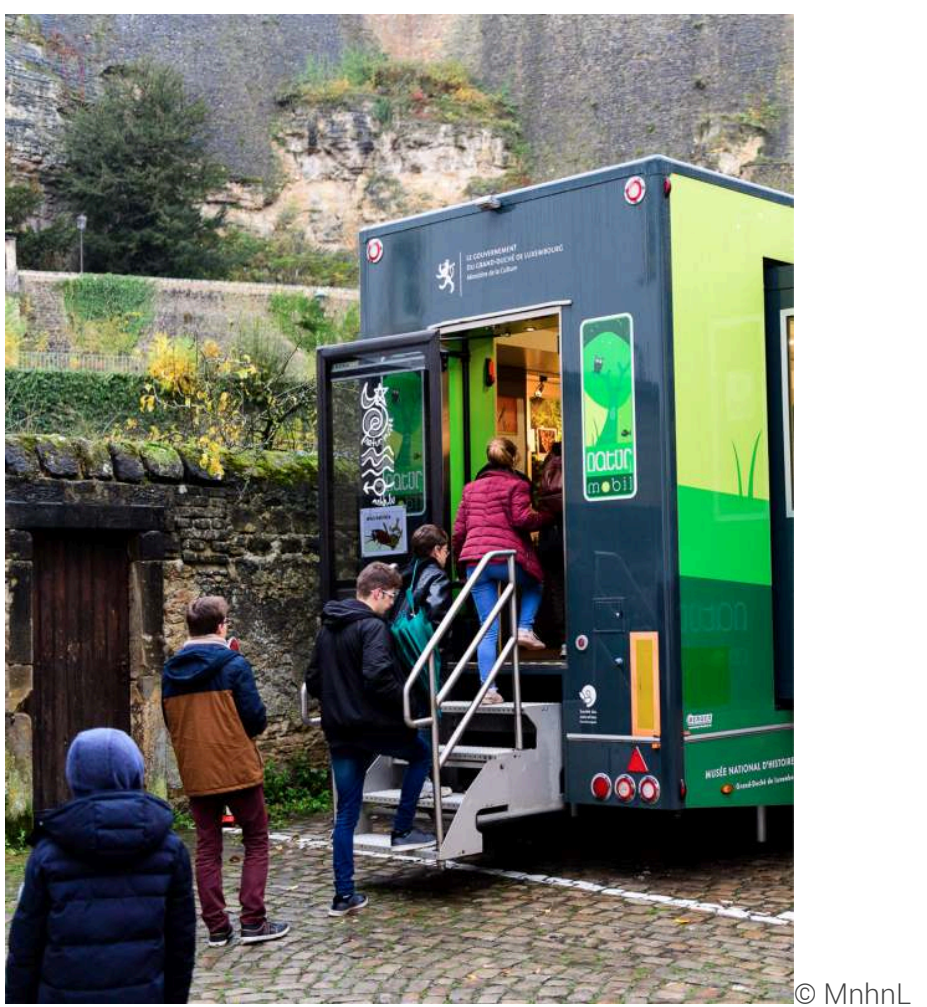




\title{
Les espaces mobiles du muséum du Luxembourg : une décentralisation réussie
}

1 Il y a 25 ans déjà que le Musée national d'Histoire naturelle du Grand-Duché du Luxembourg (MnhnL) a décidé d'investir dans un espace mobile afin de décentraliser ses activités. Localisé à Luxembourg-Ville dans un quartier au bas des vieux murs de la forteresse, le MnhnL répondait effectivement relativement bien à ses missions, mais la majorité des groupes scolaires en visite venaient de la capitale, de villages adjacents ou de localités bien connectées au réseau ferroviaire, la gare centrale étant à $15 \mathrm{mn}$ à pied du musée. Les collèges et les lycées semblaient bouder le musée.

2 Le projet de décentralisation a abouti en mai 1994: un camion ayant pour objectif de sensibiliser les habitants du pays à son patrimoine naturel sillonne depuis les routes. Arrivé à destination - le plus souvent une cour de l'école - le Natur Mobil se déploie pour se convertir en salle d'exposition et de manipulation.

Quelques années plus tard, la flotte mobile du MnhnL est renforcée par le Science Mobil qui concentre ses activités sur des sujets scientifiques que les jeunes découvrent par l'expérimentation, le questionnement et le dialogue. La physique, la chimie et la biologie y deviennent source d'émerveillement, et non de réticence.

Notons que les espaces mobiles ne sont pas les seuls outils mis en place par le MnhnL pour décentraliser ses activités. En effet, plusieurs visites animées par un guide du musée sont régulièrement proposées, que ce soit dans aux abords d'un établissement scolaire ou encore sur un site naturel proche.

\author{
Le Luxembourg en chiffres \\ Le Grand-Duché du Luxembourg \\ - Superficie : $2586 \mathrm{~km}^{2}$ \\ -Population : environs 614000 habitants \\ Les musées luxembourgeois
}

- 65 musées à travers le pays, dont 10 à Luxembourg-Ville ;

- 20 musées sont membres de l'Icom, dont 7 en ville.

Le muséum national

- 7 sections scientifiques (sciences de la vie et de la Terre)

- 1 service éducatif (visites, mobiles, etc.)

- 1 service muséographique

- Espace d'exposition : $1500 \mathrm{~m}^{2}$

- Fréquentation : 50000 visiteurs/an 


\section{Renforcer l'offre de médiation sur le territoire : motivations et complémentarité des outils de médiation}

\section{Pourquoi avoir investi dans deux espaces mobiles?}

Un musée a pour but de valoriser des collections et de conserver un patrimoine culturel (respectivement naturel) mais aussi de familiariser le public avec cette culture. L'objectif premier d'un musée face à son public est donc de l'informer, de le fasciner et de le familiariser avec les thèmes d'histoire naturelle par ses expositions, ses publications, ses visites ou ateliers, ceci en posant les premières pierres dès le plus jeune âge. Par conséquent, quoi de plus logique que d'élaborer une panoplie de dispositifs pédagogiques qui s'adressent aux élèves du Grand-Duché ?

6 Au début des années 1990, l'idée de délocaliser les activités du musée se concrétise : pourquoi ne pas aller à la rencontre des élèves? Pourquoi même ne pas pousser le projet jusqu'à l'installation d'un camion dans leur cour de récréation? Les scolaires et leurs enseignants n'auraient pas à se déplacer, ce qui représenterait non seulement un gain de temps, mais aussi une économie financière. Inutile de mentionner l'aventure que pourraient vivre les élèves en entrant dans un véhicule entièrement aménagé à l'image d'une salle éducative dans laquelle ils découvrent les animaux naturalisés de nos forêts, manipulent des loupes binoculaires ou microscopes pour partir à l'étude plus poussée de la faune et de la flore... C'est ainsi qu'est né le Natur Mobil.

7 Le pari est gagné : les élèves participent avec plaisir, s'émerveillent. Mais surtout des phrases telles que "C'était vraiment très bien. Est-ce qu'on peut revenir demain ?" provenant spontanément des enfants et les élèves qui partagent les points forts de leur visite avec leurs amis confirment que le Natur Mobil atteint ses objectifs. Face à l'écho très positif de cette formule mobile du MnhnL, qui se traduit par une augmentation des inscriptions des établissements scolaires, le besoin s'est assez rapidement fait ressentir de se plonger dans un deuxième projet du même genre, le Science Mobil, sans pour autant faire une copie de son prédécesseur ! Bien au contraire, il a permis d'agrandir le champ de sensibilisation aux sciences dites «dures» et aux technologies et de s'adresser de manière renforcée à un public encore un peu réticent, les collèges et les lycées. Alors que les sujets traités par le Natur Mobil restent axés sur la nature nous environnant, donc plutôt sur l'éveil aux sciences, le Science Mobil veut intégrer plus de chimie et physique dans ses activités. La première exposition du Science Mobil avait ainsi pour sujet les énergies alternatives avec par exemple des manipulations autour de la physique et la technologie des éoliennes.

8 C'est ainsi que depuis 2002 les régions et communes éloignées de Luxembourg-Ville ont accès aux programmes éducatifs du MnhnL dans une formule hors les murs, adaptée aux problématiques des territoires ruraux isolés. 
Dès les premiers instants, les structures Mobil éveillent la curiosité des enfants. Ici le Natur Mobil prêt à accueillir ses jeunes visiteurs.

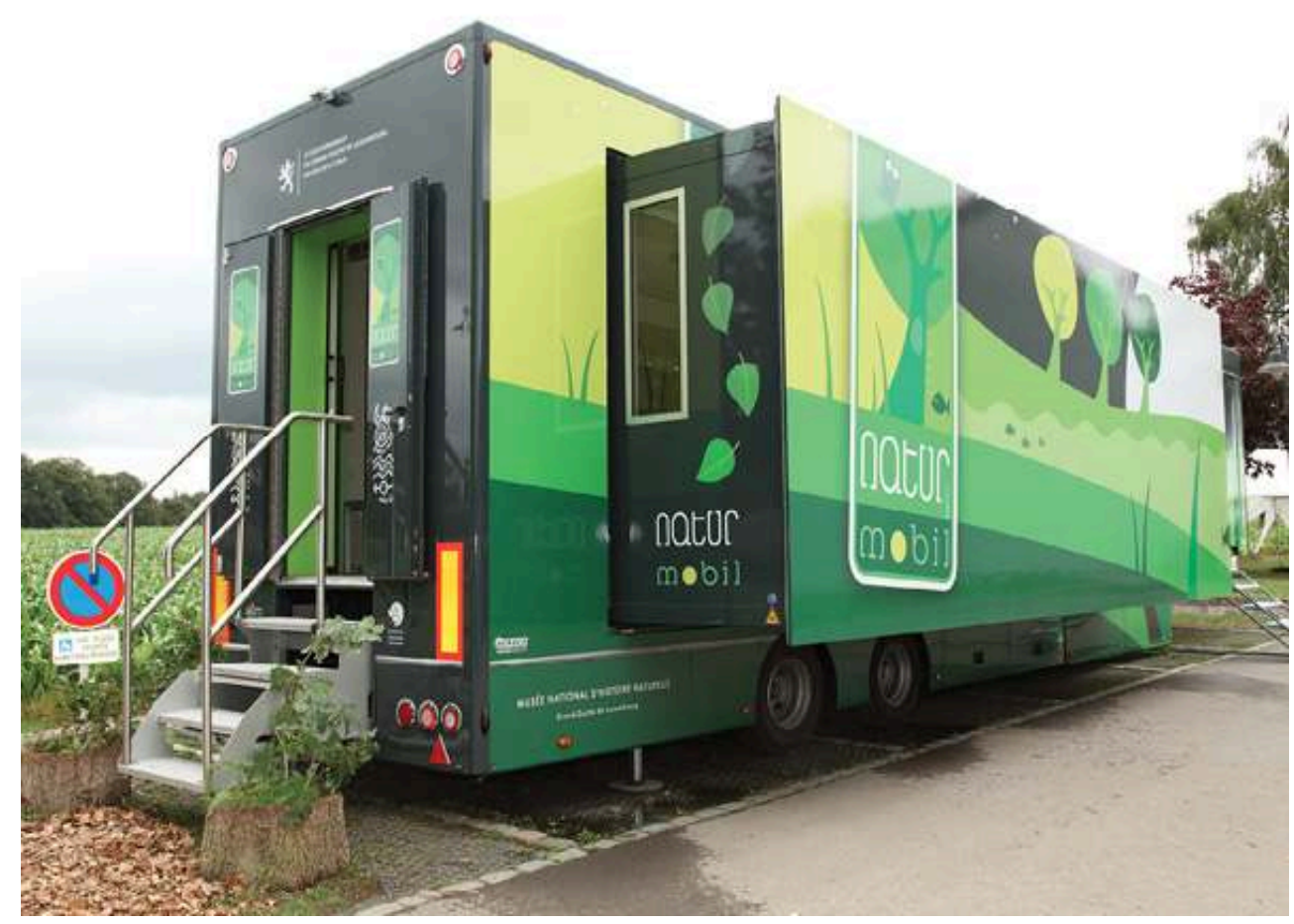

(c) MnhnL

\section{Zoom sur le Natur Mobil}

Difficile pour les habitants de ne pas remarquer le Natur Mobil: ses couleurs et son stylisme permettent de dévoiler les thématiques abordées - la découverte et la conservation du patrimoine naturel de notre pays - et rendent la structure rapidement identifiable par les usagers réguliers.

Le Natur Mobil s'adresse principalement aux élèves de l'enseignement fondamental (4-11 ans), mais accueille aussi avec plaisir ceux de l'enseignement modulaire, pourtant plus rares. Tous, peu importe leur âge, partent activement à la découverte de leur entourage naturel souvent méconnu. La plupart des unités pédagogiques comprennent une sortie en nature (dans une forêt, au bord d'un étang...) afin de récolter des échantillons, suivie d'une séance à l'intérieur de l'espace mobile. Quoi de plus naturel et de plus passionnant pour découvrir la faune et la flore de la forêt que l'exploration et la recherche d'espèces locales. Les enfants sont stimulés par la découverte des espèces rencontrées et leur habitat diversifié. Ils sont d'autant plus impatients de rejoindre le Natur Mobil pour prolonger l'étude de leurs «trouvailles " grâce à des instruments scientifiques. Une occasion aussi de poursuivre les explications sur ce qu'ils ont observé sur le terrain. Les enfants sont fiers de pouvoir ensuite partager avec leurs proches l'aventure qu'ils viennent de vivre. Pour les enseignants les activités du Natur Mobil représentent souvent un complément idéal et surtout très vivant à l'éveil aux sciences et aux sciences naturelles du programme de l'enseignement fondamental. L'atout du Natur Mobil est certainement le matériel didactique (microscopes, loupes binoculaires, animaux naturalisés, panneaux et vitrines amovibles...) faisant souvent défaut dans les écoles. 
Intérieur du Natur Mobil lors de l'activité sur les secrets de la digestion.

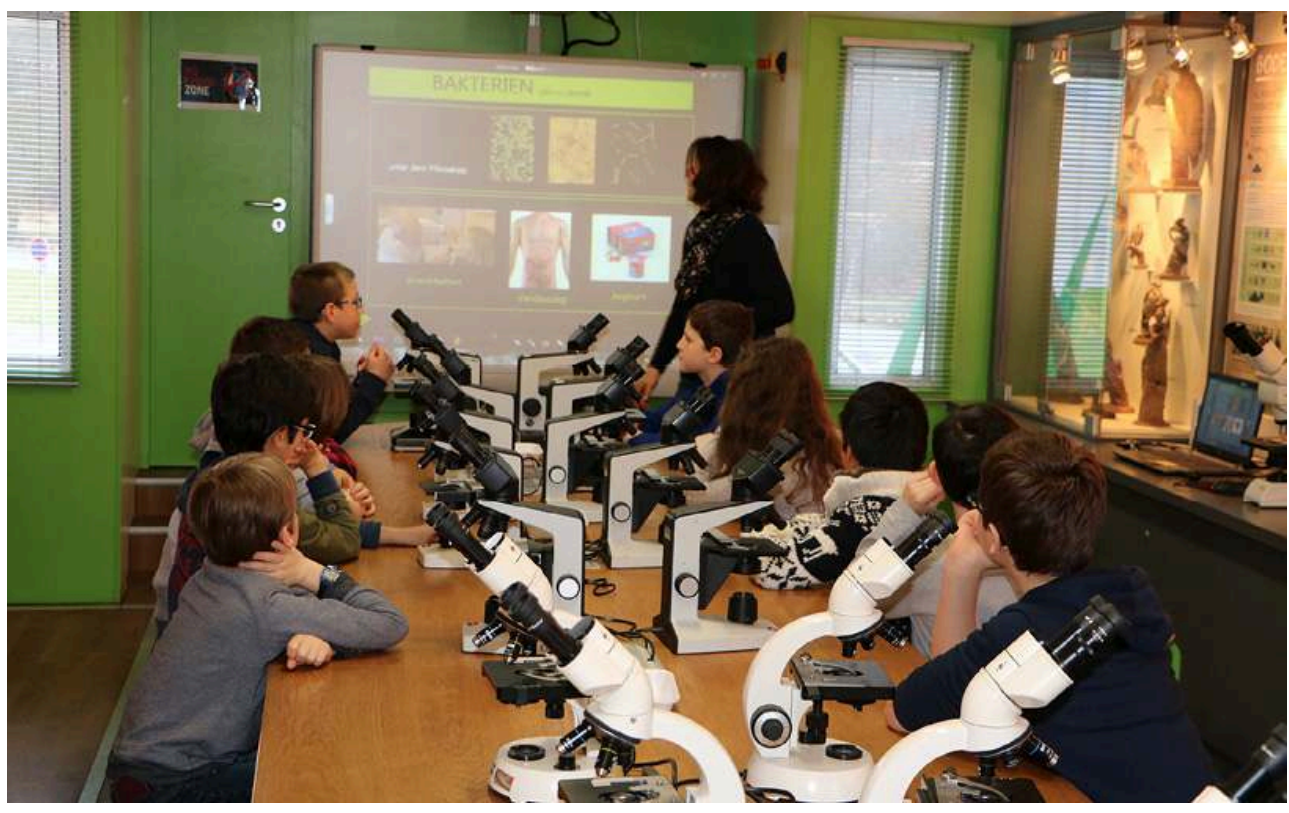

(C) MnhnL

11 Les activités proposées dépendent des saisons et peuvent être spécialement adaptées à la région visitée pour approfondir les sujets d'intérêt local. Le Natur Mobil est le moyen idéal de porter les campagnes de sensibilisation du MnhnL au cœur même des villages et des communes. Il permet d'impliquer activement les jeunes sur le terrain et ceci dans les alentours directs de leur lieu de vie.

\section{Les activités du Natur Mobil}

- À la découverte de la forêt

- Eau propre ou polluée

- La vie dans le sol

- Le loup

- Les secrets de la digestion

- Microscopie

- Quand la vie s'est installée sur notre terre

- Traces et indices de la vie

-Vie dans les prés

-Vie dans l'étang

- Vie sous la loupe 


\section{Zoom sur le Science Mobil} grand public qui souhaite savoir ce que ce «monstre » cache dans son ventre. Mais bien que ses portes soient ouvertes à tous, le Science Mobil s'adresse surtout aux élèves de l'enseignement fondamental (9-11 ans) et du cycle inférieur de l'enseignement secondaire (12-15 ans). Son objectif est de donner un aperçu passionnant des sciences en dépassant les cours magistraux courants et inévitables dans l'enseignement. Peu importe les intérêts d'un élève, il est toujours possible de susciter sa curiosité pour un sujet qui lui est inconnu ou qu'il juge trop compliqué ! La glace est généralement brisée lorsque l'élève est complètement impliqué et qu'il vit activement le moment. C'est justement sur ce constat que se basent les activités du Science Mobil.

Le Science Mobil prêt à prendre la route.

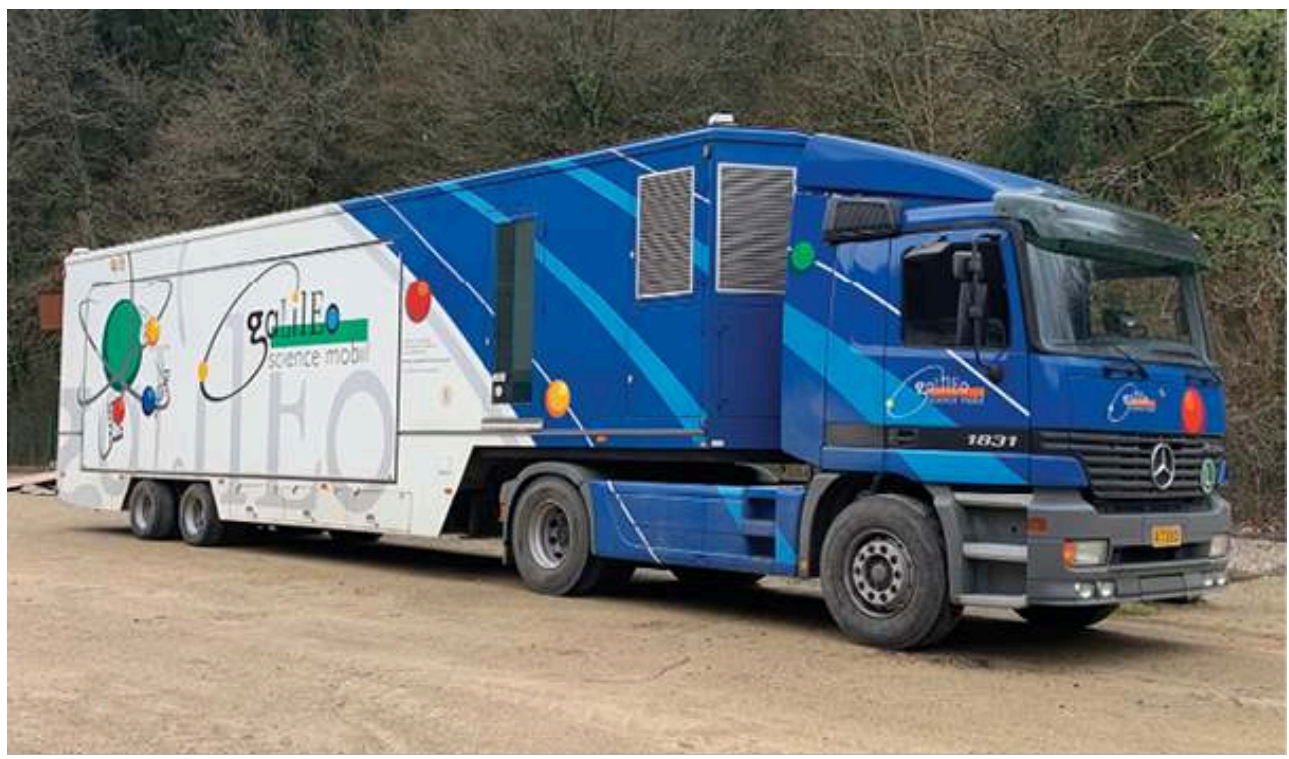

(C) MnhnL

Quitter sa salle de classe pour rentrer dans un semi-remorque déployé en un espace de $42 \mathrm{~m}^{2}$ représente déjà pour certains élèves une expérience mémorable. Ceci se lit sur leurs visages lorsqu'ils en découvrent l'intérieur et se confirme lorsqu'un élève revient quelques années plus tard en s'exclamant « Mais j'étais déjà ici, on a parlé de... ! ». Ensuite l'aventure continue pendant deux heures au cours desquelles s'alternent discussions, explications et petites expériences. Prenons l'exemple de l'unité «Quel rayonnement! » de l'exposition en cours (voir encadré) pour donner un aperçu de ce que vivent les élèves. Après avoir cherché des exemples d'objets émettant de la lumière (ampoules, soleil, vers luisants, lave, feu, etc.) les élèves sont invités à les classer selon des critères qu'ils définissent. Ils découvrent ensuite la classification "officielle " (incandescence, fluorescence, bioluminescence...) et ses caractéristiques pour y regrouper au fur et à mesure les exemples cités lors de l'entrée en matière. Les élèves remarquent qu'instinctivement, bien que leurs critères diffèrent parfois de ceux des scientifiques, leur classement était relativement correct mais surtout qu'ils peuvent rapidement utiliser des connaissances scientifiques fraîchement acquises. Les petites expériences, réalisées en binômes sont indispensables dans les unités du Science Mobil! 
Comment mieux expliquer aux élèves les caractéristiques de la fluorescence qu'en leur demandant de saupoudrer un peu de fluorescéine dans de l'eau pour ensuite illuminer le mélange? Les exclamations telles "Ouah » ou "Est-ce que je peux prendre une photo?» fusent. L'intérêt et la curiosité des élèves sont alors captés, ils comprennent et nous expliquent le principe de base et sont ouverts pour des explications plus compliquées. Cette méthode alternant travail en groupe, en binôme, petites manipulations et explications scientifiques constitue un bon mélange permettant de répondre aux objectifs du Science Mobil: éveiller l'intérêt des jeunes pour les sciences; montrer qu'elles sont accessibles à condition d'être curieux; et prouver que les principes scientifiques sont omniprésents au quotidien.

Les expositions du Science Mobil

- Les énergies alternatives et renouvelables

2002-2005

- Les astuces de la nature

2005-2008

- Le sens des sens

2008-2012

- Le lait, le savon, les vêtements... sous la loupe!

2012-2016

- La lumière dans tous ses éclats

à partir de 2016

- Nouvelle exposition sur le thème de l'évolution

en préparation 
Intérieur du Science Mobil équipé de l'exposition La lumière dans tous ses éclats.

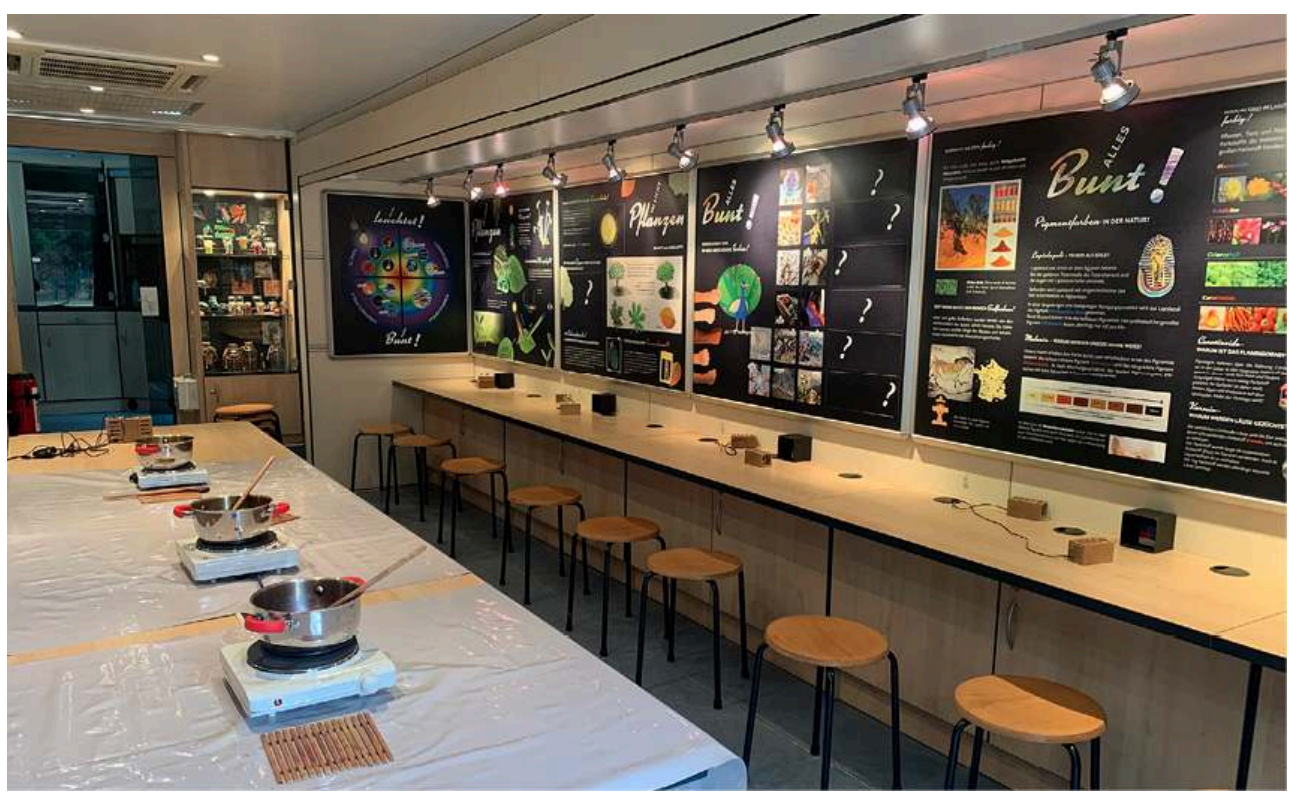

(C) MnhnL

\section{L'écho de la population luxembourgeoise face aux espaces mobiles du MnhnL}

Les paris des deux espaces mobiles sont gagnés! Effectivement ils se déplacent à travers tout le pays, de la plus petite à la plus grande commune, et ceci avec pour seule pause les vacances scolaires. Dès les premiers instants, ils suscitent la curiosité des enfants. Leur émerveillement continue généralement lorsqu'ils entrent dans le véhicule et partent à la découverte du patrimoine ou des sciences avec l'animateur.

Les chiffres sont très parlants quant au succès des deux semi-remorques. Deux mois après la rentrée, le planning du Science Mobil est déjà complet pour toute l'année scolaire. Le succès du Natur Mobil pose un véritable défi: le nombre croissant de demandes induit le risque de ne pas les satisfaire toutes et de décourager les gens à tenter leur chance. La liste d'attente s'allonge et certaines écoles doivent patienter deux à trois ans avant de pouvoir profiter de ses activités. Le passage des groupes se fait pourtant au rythme de deux à trois classes par jour pour le Science Mobil et d'une à deux classes pour le Natur Mobil! 
Récolte d'échantillons dans la forêt. Un champignon dans la main gauche, un arthropode dans la main droite.

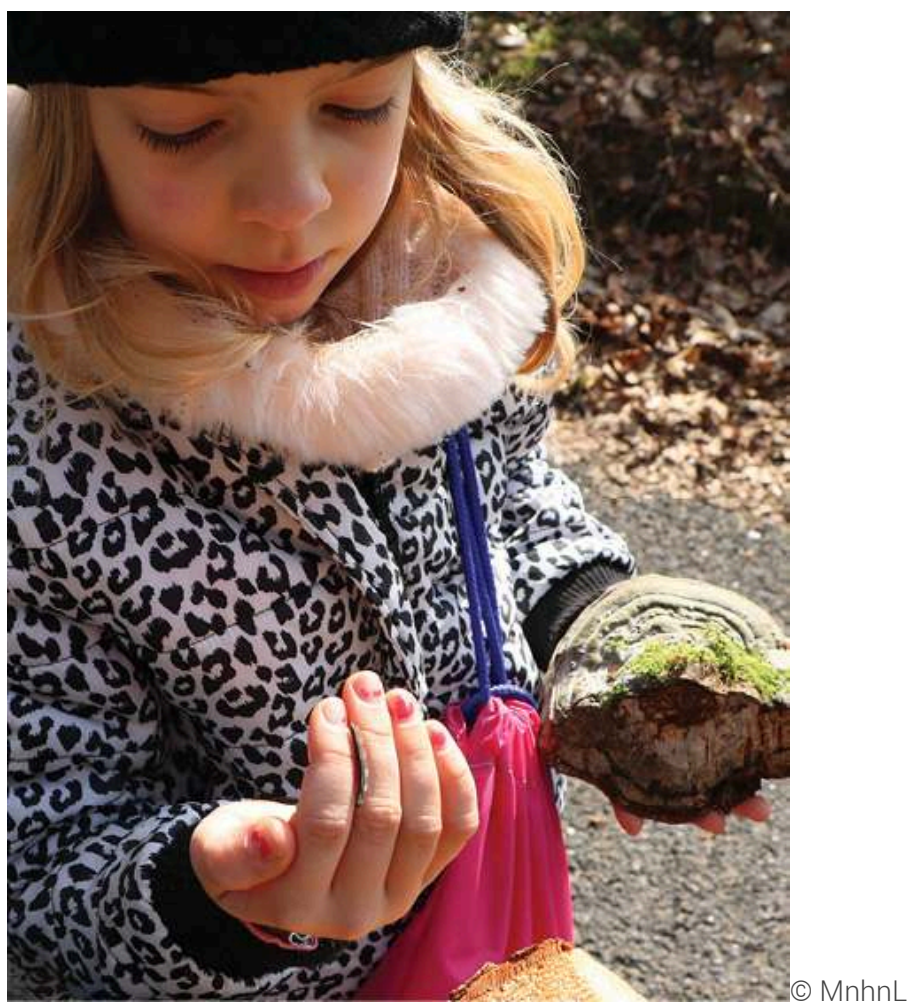

En 26 ans, le Natur Mobil a accueilli 147346 visiteurs avec une moyenne annuelle de 4 150 personnes par an sur les dix dernières années. Le nombre décroissant s'explique par le fait que le Natur Mobil ne participe plus que sporadiquement à des portes ouvertes. La moyenne de visiteurs des dernières années se limite donc aux élèves participant à ses activités courantes et aux jeunes participant à des activités de loisirs organisées avec d'autres équipes du service éducatif du MnhnL.

Le Science Mobil sillonne le pays depuis 17 ans. Cela représente 103815 curieux de tout âge qui se sont adonnés aux sciences. Le pic de visiteurs très important en 2003 est dû au grand nombre de portes ouvertes organisées lors de toutes sortes de manifestations dans le but de présenter le Science Mobil. Depuis 2006 la moyenne annuelle atteint 5700 visiteurs et se compose de différents publics cibles.

\section{Les limites et évolutions thématiques des espaces mobiles}

Il n'est pas toujours facile pour les établissements scolaires de trouver un emplacement adéquat dans la cour de récréation, les camions nécessitant un emplacement assez grand, plat et accessible, mais surtout un raccordement électrique jour et nuit de 380 volts. Ces petits obstacles sont pourtant généralement surmontés, même si parfois le camion n'est installé qu'à proximité de l'école. Le Natur Mobil doit en plus régulièrement organiser un transport entre le site de découverte et le camion. Mais là aussi des solutions sont toujours trouvées. 
Nombre de visiteurs du Natur Mobil et du Science Mobil depuis leurs débuts respectifs.

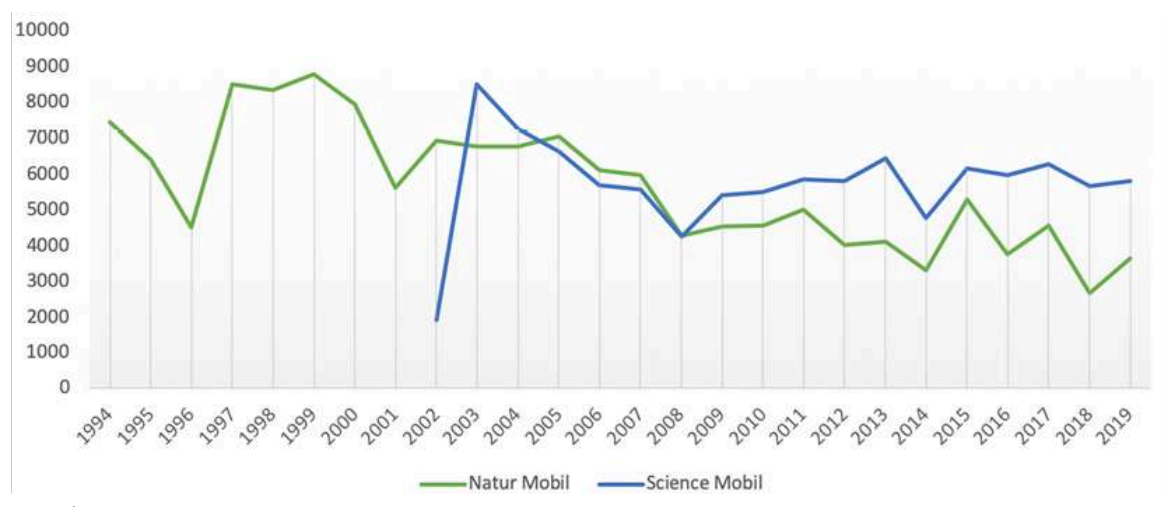

(c) MnhnL

Il est relativement rare que les élèves de l'enseignement secondaire profitent de l'offre du Natur Mobil. Pour que les élèves aient le temps de découvrir un habitat sur le terrain et ensuite analyser quelques données dans le semi-remorque, trois heures doivent être imputées sur le programme scolaire. Ceci cause certains problèmes d'organisation des cours dans les établissements puisque l'enseignant change généralement de classe toutes les heures, il doit aussi trouver un remplaçant et "perd» de précieuses heures de cours dans ses classes.

En réaction au besoin de s'adapter aux changements de la société, l'équipe du Natur Mobil a décidé depuis quelques années de mettre davantage l'accent sur la découverte et l'exploration de la nature et moins sur la transmission d'informations plus poussées sur le patrimoine naturel. En effet, vu la société multiculturelle du Luxembourg, de nombreux élèves ne découvrent la langue luxembourgeoise que lors de leur scolarisation. Ceci induit des problèmes de communication surtout dans les classes inférieures. De plus, les enfants sortent de moins en moins dans la nature pour la découvrir. L'exploration de l'environnement naturel devient donc primordiale, au détriment peut-être de la transmission de connaissances plus approfondies.

Les publics du Science Mobil.

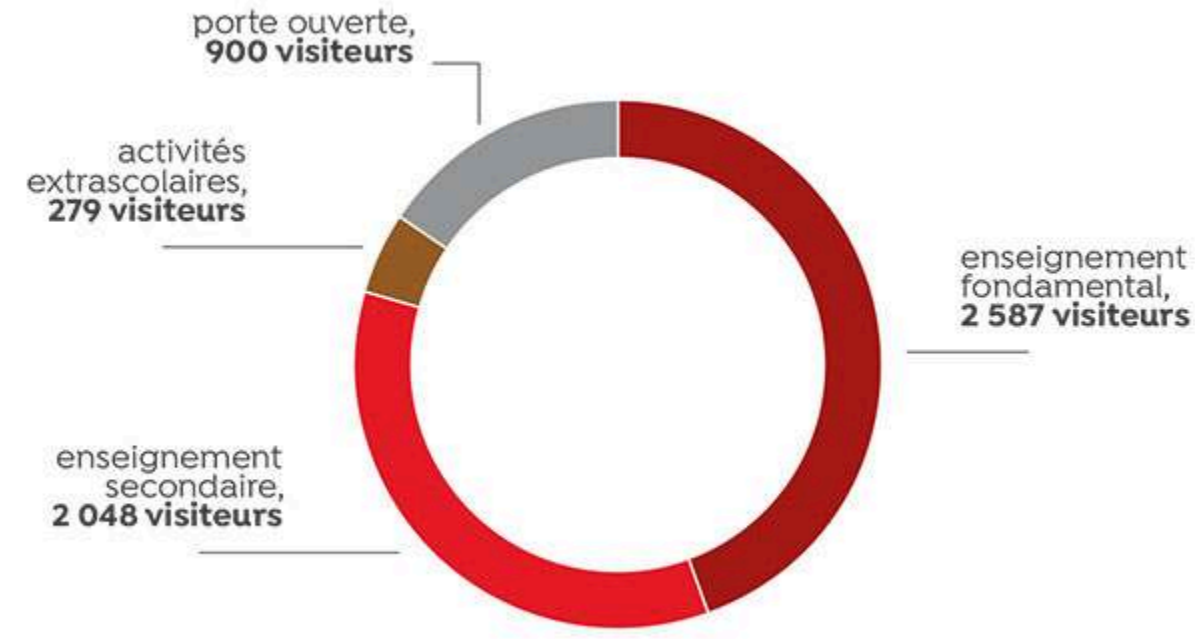


21 classe pour permettre de travailler avec la qualité espérée (répondre aux questions de chacun, accompagner au mieux les binômes lors des manipulations...). C'est la raison pour laquelle nous demandons aux enseignants de scinder leur classe en deux groupes si elle compte plus de 21 élèves. Ceci touche $15 \%$ des classes scolaires. L'idée est relativement bien acceptée! La nécessité de scinder les classes en deux, demande un plus en séances pour les animateurs et réduit le nombre d'élèves par année, mais la qualité de l'activité se trouve fortement augmentée. couvrent cependant que partiellement les frais du personnel. Par contre, ils incitent les animateurs à des prestations de qualité et encouragent les enseignants à intégrer utilement les séances dans l'éducation des jeunes.

\section{Conclusion} sensibilisation de leur public et la transmission du savoir. Pour ce faire, les équipes remettent continuellement leurs méthodes en question avec pour souci de les améliorer. Face au Covid 19 nous avons par exemple décidé de restructurer nos unités. Effectivement, bien qu'ils soient grands, l'espace des mobiles est trop restreint pour accueillir les classes scolaires dans le respect des conditions d'hygiène. Nous avons remodelé nos unités pour pouvoir passer plus de la moitié de l'activité à l'extérieur des véhicules mais tenons cependant à maintenir la conclusion et/ou l'introduction en intérieur, pour ne pas enlever l'aventure que vivent les élèves en rentrant dans le Science Mobil et le Natur Mobil. En cas de très mauvais temps, nous avons décidé de nous déplacer avec notre matériel pédagogique dans la salle de classe du groupe inscrit ou dans une salle nous étant réservée pour la semaine. Cette seconde formule n'inclut malheureusement pas les mobiles, mais nous permet de continuer nos activités de vulgarisation des sciences et de transmission de notre passion. Nous espérons évidemment pouvoir, dans un futur proche, accueillir les élèves dans ce décor unique qu'offrent les mobiles.

26 Pour diversifier son offre, le Natur Mobil élabore régulièrement de nouvelles unités, celle sur le loup étant la plus récente. Le Science Mobil quant à lui compte remplacer son exposition actuelle par une nouvelle exposition début 2021. Celle-ci sera axée sur l'évolution. 


\section{RÉSUMÉS}

Offre originale pour un muséum, les espaces mobiles développés par le Musée national d'Histoire naturelle $d u$ Luxembourg permettent d'assurer la mission de médiation scientifique sur l'entièreté du territoire, y compris dans les zones rurales ou éloignées. Une opportunité de toucher et de conquérir un nouveau public.

\section{INDEX}

Mots-clés : exposition itinérante, animation scientifique, muséum d'histoire naturelle

\section{AUTEURS}

\section{SANDRINE AMANN}

Coordinatrice du Science Mobil au Musée national d'Histoire naturelle du Grand-Duché du Luxembourg

Sandrine.Amann@mnhn.lu

\section{ALAIN FABER}

Directeur du Musée national d'Histoire naturelle du Grand-Duché du Luxembourg Alain.Faber@mnhn.lu 